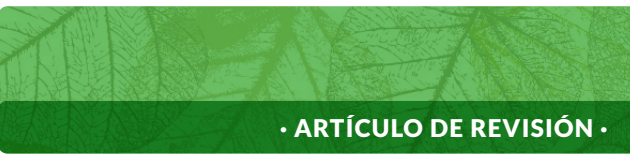

\title{
Aplicación de altas presiones y otras tecnologías en frutas como alternativa de tratamientos térmicos convencionales*
}

\section{Application of high pressures and other technologies in fruits as alternative of conventional thermal treatments}

\author{
FIGUEROA-SEPÚLVEDA, KATHERINE'; CASTILLO-ROBLES, NELLY-ZARITH'²; \\ MARTÍNEZ-GIRÓN, JADER ${ }^{3}$
}

Historial del Artículo

Recibido para evaluación: 5 de Junio 2019.

Aprobado para publicación: 11 de Septiembre 2019.

* $\quad$ Título del proyecto de origen: "Tecnologías Emergentes en Agroindustria de Frutas y Hortalizas, Resultados de la Actividad Académica y Científica de Tendencias e Innovaciones Agroindustriales (5000912)". Financiación: Universidad Nacional de Colombia-Sede Palmira. Culminación: 31 Octubre de 2020.

1 Universidad Nacional de Colombia Sede Palmira, Facultad de Ingeniería y Administración, Joven Investigadora. Ingeniera Agroindustrial. Palmira, Colombia. https://orcid.org/0000-0002-5488-840X

2 Universidad Nacional de Colombia Sede Palmira, Facultad de Ingeniería y Administración, Joven Investigadora. Ingeniera Agroindustrial. Palmira, Colombia. https://orcid.org/0000-0002-7345-4500

3 Universidad del Valle Sede Palmira. Doctorando en Ciencia y Tecnología en Alimentos. Palmira, Colombia. https://orcid. org/0000-0003-4191-5445

Correspondencia: jader.martinez@correounivalle.edu.co

Como citar este artículo: FIGUEROA-SEPÚLVEDA, KATHERINE; CASTILLO-ROBLES, NELLY-ZARITH; MARTÍNEZ-GIRÓN, JADER. Aplicación de altas presiones y otras tecnologías en frutas como alternativa de tratamientos térmicos convencionales. Biotecnología en el sector agropecuario y agroindustrial, v. 19, n. 2, 2021, p. 271-285. Doi: https://doi.org/10.18684/bsaa.v19.n2.2021.1772 


\section{RESUMEN}

En la actualidad la industria alimentaria busca ofrecer productos alimentarios frescos pero a su vez que tengan una vida útil prolongada. Existen varias técnicas que requieren tratamiento térmico las cuales permiten aumentar la vida útil de los alimentos pero estas pueden afectar la calidad final del producto. La alta presión hidrostática y la homogeneización por altas presiones son nuevas técnicas no térmicas que permiten actuar sobre los alimentos a temperaturas de proceso bajas alargando la vida útil y conservando la calidad nutricional. La alta presión hidrostática se ha aplicado en diferentes frutas mínimamente procesadas para conservar su frescura y propiedades nutricionales entre ellas: duraznos, mango, cerezas, fresas, pulpa de naranja, melón, chirimoya y las aceitunas donde se ha determinado que esta técnica conserva mejor las propiedades de estos materiales vegetales respecto a tratamientos térmicos convencionales. En cuanto a la homogeneización por alta presión se ha aplicado principalmente en jugos de manzana, fresa, mango, sandía, toronja y zumo de naranja obteniendo una buena inactivación de microorganismos y una mejor conservación de compuestos bioactivos, sin afectar las propiedades nutricionales y funcionales.

\section{ABSTRACT}

Currently, the food industry seeks to offer fresh food products but also have a long shelf life. There are several techniques that need heat treatment which allow prolonging the shelf life of food but these can affect the final quality of the product. High hydrostatic pressure and homogenization by high pressure are new non-thermal techniques that allow to act on food at low process temperatures, lengthening the shelf life and preserving the nutritional quality. High hydrostatic pressure has been applied to different minimally processed fruits to preserve their freshness and nutritional properties, including peaches, mango, cherries, strawberries, orange pulp, melon, custard apple and olives where it has been determined that this technique better preserves the properties of these plant materials compared to conventional heat treatments. Regarding high pressure homogenization, it has been applied in apple, strawberry, mango, watermelon, grapefruit and orange juice juices, obtaining a good inactivation of microorganisms and a better preservation of bioactive compounds that improve nutritional and functional properties.

\section{INTRODUCCIÓN}

En la industria alimentaria es indispensable el uso de técnicas que permitan prolongar la vida útil de los alimentos, como lo son: la refrigeración, congelación, secado, deshidratación, ahumado, entre otras. Siendo la pasteurización y la esterilización las técnicas más usadas para inactivar enzimas y microorganismos alterantes en productos alimenticios, los cuales deterioran los alimentos (Ruiz et al., 2021). Sin embargo, la pasteurización y la esterilización no sólo extienden la vida útil del alimento sino que también pueden afectar la calidad del producto, por lo que se requiere la

\section{PALABRAS CLAVE:}

Calidad; Compuestos bioactivos; Conservación; Enzimas;

Esterilización; Microorganismos; Pasteurización; Procesamiento;

Temperatura; Vegetal.

\section{KEYWORDS:}

Quality; Bioactive compounds; Conservation; Enzymes; Sterilization; Microorganisms; Pasteurization; Processing; Temperature; Vegetable. 
implementación de nuevas técnicas de conservación principalmente para compuestos termosensibles (Hradecky et al., 2017). La pasteurización térmica principalmente es utilizada para la fabricación de productos alimenticios microbiológicamente seguros, mediante la reducción o inactivación del recuento microbiano (Deshaware et al., 2019), pero debido a la alta temperatura que se requiere, esta puede afectar las propiedades organolépticas y nutricionales en algunos alimentos en comparación con las tecnologías no convencionales (Cheng et al., 2020). Por su parte, la esterilización es considerada como una de las técnicas más eficaces en la preservación de alimentos ya que puede proporcionar la inactivación casi completa de microorganismos incluyendo las esporas, lo que conlleva a que los productos tengan una mayor vida útil (Hradecky et al., 2017; Giraldo-Gil et al., 2020) pero esta al igual que la pasteurización puede en algunos casos afectar las propiedades nutricionales principalmente de los compuestos antioxidantes incluyendo las vitaminas e inducir en cambios de color, sabor y textura los cuales afectan la calidad sensorial del alimento; incluso se pueden llegar a formar subproductos indeseables durante el procesamiento que afectan la calidad del producto final (Hradecky et al., 2017; Martín-Vertedor et al., 2020). Debido a lo anterior, la industria alimentaria está en continua búsqueda de tecnologías emergentes que permitan reducir microorganismos patógenos, como bacterias y hongos los cuales reducen el tiempo de conservación de los alimentos y aumentan el riesgo de enfermedades transmitidas por alimentos (ETA) (Dai et al.,2020), además de sustituir las técnicas tradicionales como las mencionadas anteriormente, por nuevas metodologías que permitan conservar al máximo las características nutricionales y sensoriales de los alimentos. Lo anterior, teniendo en cuenta que la demanda de los consumidores actuales está orientada al consumo de productos nutritivos, funcionales y fáciles de preparar, como por ejemplo los jugos de frutas enriquecidos con minerales y vitaminas (Agudelo-Martínez et al., 2020).

En la actualidad, las técnicas más investigadas que no requieren de tratamientos térmicos fuertes son: la alta presión hidrostática, campos eléctricos pulsados, radiación ionizante, ultrasonido, microondas, plasma frío, entre otras. Las cuales son capaces de inactivar microorganismos a temperatura ambiente o temperaturas no muy elevadas impidiendo defectos ocasionados por el calor (Cheng et al., 2020; Maniglia et al., 2021) con el fin de producir alimentos más seguros y minimizar las pérdidas y desperdicios ocasionados por el deterioro de los alimentos, en especial de frutas y hortalizas las cuales son muy perecederas. En tal sentido, esta revisión presenta la aplicación de las altas presiones hidrostáticas y de homogenización como mecanismo en la inactivación de microorganismos y la preservación de compuestos bioactivos en productos alimenticios frescos y procesados a partir de frutas.

\section{DESARROLLO DEL TEMA}

\section{Altas presiones hidrostáticas}

En la tecnología de altas presiones hidrostáticas (APH) la presión es aplicada de manera uniforme y al instante, la variación de la temperatura es poca a medida que aumenta la presión, evitando que la del alimento se deforme o alcance elevadas temperaturas, lo cual no modifica las propiedades sensoriales de los alimentos (Rios-Corripio et al., 2020). La inactivación de los microorganismos es causada por el daño y/o ruptura de la membrana, dificultando el transporte de nutrientes y la eliminación de los residuos de células, desnaturalización de proteínas, disminución del pH intracelular, inactivación de enzimas y disminución del tamaño de partícula como por ejemplo en sistemas coloidales y emulsiones alimentarias (Gharibzahedi-Taghi et al., 2019; Huang et al., 2020). La tecnología de altas presiones hidrostáticas consiste en la aplicación de una presión alta en el orden de MPa, habitualmente en un intervalo de 100-600 MPa o superior, transmitida por un líquido acuoso, que generalmente es agua, en un sistema cerrado (Rios-Corripio et al., 2020; Huang et al., 2020), la cual se puede desarrollar durante un corto tiempo de exposición de la muestra, por lo general de 5-10 min (Perdomo-Lamilla et al., 2020; Hu et al., 2021). El efecto de la APH no solo varía dependiendo del tipo de equipo, sino que puede variar de acuerdo con las condiciones de procesamiento (presión, tiempo de retención, pH, temperatura), el estado fisiológico, la matriz y la forma de los alimentos (entero, trozos, puré o zumo) (Wolbang et al., 2008; Andrés et al., 2016). 
Esta tecnología puede actuar en frío, a temperatura ambiente o a temperaturas de proceso por lo general $<60$ ${ }^{\circ} \mathrm{C}$ (Perdomo-Lamilla et al., 2020) lo que hace que se conserve el valor nutricional y la calidad final del producto, debido a que los compuestos de bajo peso molecular, como los responsables del olor y sabor, pigmentos y algunas vitaminas no se alteran, además la presión no afecta la unión covalente de estas, también mantiene la textura y evita la degradación de antioxidantes (Meng et al., 2019; Gómez-Maqueo et al., 2020) otorgando características frescas las cuales conducen a garantizar la calidad, seguridad (inocuidad) y una mayor vida de anaquel en los productos alimenticios. Adicionalmente, esta tecnología podría proporcionar a los consumidores alimentos en los que se minimice o elimine el uso de aditivos químicos, permitiendo la adición de cultivos prebióticos y la elaboración de productos fermentados (Marcos et al., 2007), como respuesta a la demanda de los consumidores por alimentos procesados que sean más sanos e innovadores. Estas ventajas han hecho que se implemente esta tecnología en varias industrias de procesamiento de alimentos, principalmente en la agroindustria de frutas y hortalizas (Manassero et al., 2019). Dado lo anterior, esta tecnología emergente ha sido aprobada por el Departamento de Agricultura de Estados Unidos (USDA), la Administración de Alimentos y Medicamentos (FDA), el Codex Alimentarius (CA) y la Entidad de Salud y Servicios Humanos (HHS) (Bover-Cid et al., 2011; USDA, 2012; Huang et al., 2020). Otra de sus ventajas es que puede ser aplicada como una medida de conservación final en alimentos envasados, como una etapa de pasteurización en frío directamente en el envase del producto, ofreciendo un nivel de seguridad microbiológica que permita una inocuidad garantizada a los productos listos para el consumo (Bover-Cid et al., 2011).

\section{Homogeneización por alta presión}

La homogeneización por alta presión (HAP) es una operación cuyo principio es similar a la homogenización convencional, en la que un fluido es forzado a través de un pequeño orificio (válvula o boquilla) (Ye and Harte, 2014) por una presión muy alta, luego la presión se reduce de forma rápida e instantánea, el efecto de atomización causa una combinación de fenómenos físicos (Jin et al., 2015). Dichos fenómenos son la caída de presión, cavitación, tensión de cizallamiento, turbulencia y colisión. Por lo que la homogenización por alta presión logra la reducción o inactivación de microorganismos, sin afectar el valor nutricional de los productos alimenticios (Suárez-Jacobo et al., 2011). El nivel de inactivación depende de la naturaleza del microorganismo y la presión aplicada. En general, las bacterias gram positivas son más resistentes a HAP, mientras que las bacterias gram negativas son más sensibles a HAP, seguido de levaduras y mohos (Calligaris et al., 2012). La HAP es un proceso continuo el cual reduce notablemente la carga microbiana en comparación con otras técnicas comunes de uso agroindustrial (Dumay et al., 2013). Los fenómenos físicos que se dan en la homogeneización conducen a un aumento de temperatura, sin embargo la homogeneización por alta presión se puede considerar como una tecnología no térmica, ya que el aumento de temperatura ocasionado por la fricción en la válvula es mínimo (aproximadamente $0,2^{\circ} \mathrm{C}$ ) (Maresca et al., 2011). A su vez, esta técnica permite procesar alimentos fluidos en continuo, lo cual es una ventaja que en conjunto con lo anteriormente mencionado la hace una alternativa al tratamiento térmico de pasteurización convencional (Velázquez-Estrada et al., 2012). Esta técnica además de inactivar microorganismos, se emplea para disminuir el tamaño de partícula, estabilizar emulsiones, modificar propiedades funcionales en proteínas y mejorar las propiedades reológicas y de textura en productos alimenticios (Liu and Kuo, 2016; Gharibzahedi-Taghi et al., 2019) siendo la agroindustria de frutas y hortalizas considerada como un área viable para la aplicación de tratamientos por HAP, con el fin de lograr la reducción de la carga microbiana, preservando los atributos de calidad de los productos frescos (Maresca et al., 2011). Finalmente, dependiendo del rango mínimo de presión y el efecto de ciclos aplicados la tecnología emergente se denominará homogenización por alta presión 150-200 MPa o en su defecto homogeneización de ultra alta presión 350-400 MPa (Guan et al., 2016).

\section{Aplicación de altas presiones en frutas mínimamente procesadas, jugos y zumos}

Frutas mínimamente procesadas. En la actualidad los consumidores cada vez son más exigentes a la hora de comprar una fruta mínimamente procesada; buscando que sean libres de aditivos, con una apariencia fresca y con una vida útil prolongada. Sin embargo, esta es alterada por el procesamiento de tejidos. El procesamiento por alta presión ha evidenciado tener éxito en mantener la frescura de los alimentos (Denoya et al., 2016) alar- 
gando la vida útil. Entre las aplicaciones en frutas se encuentra el uso de la tecnología de altas presiones en duraznos (Prunus persica), donde se ha evaluado como afecta las propiedades del fruto como la textura, parámetros cromáticos de color $\mathrm{CIEL}^{*} \mathrm{C}^{*}$ h, actividad enzimática de la polifenoloxidasa y contenido de etanol (Denoya et al., 2017). Estas características también se han evaluado en cubos de duraznos donde se han sometido a diferentes tratamientos acompañando la aplicación de HAP con vacío y se ha encontrado que a pesar de que se presenta ablandamiento en el tejido, se conserva mejor el color y la textura durante los días de almacenamiento. En algunas variedades como la Romea se evita el pardeamiento enzimático, debido a que se da una inhibición de las enzimas causantes del deterioro (Denoya et al., 2016), aumentando las concentraciones de Vitamina C (ácido ascórbico) y fenoles totales, lo cual es un aspecto positivo para el desarrollo de productos de alta calidad a partir de frutas. Por otro lado, otros tratamientos que se han realizado con altas presiones acompañado de vacío han sido aplicados a trozos de duraznos pretratados (Denoya et al., 2017) con una solución de ácido ascórbico y ácido cítrico para inhibir el pardeamiento enzimático evidenciando que hay una disminución en la actividad de la polifenoloxidasa y de etanol conservando los duraznos frescos durante 21 días a $10^{\circ} \mathrm{C}$. Otro pretratamiento que se puede aplicar es ácido ascórbico más lactato de calcio (Miguel-Pintado et al., 2013) influyendo en la disminución del $\mathrm{pH}$ mientras que el contenido de sólidos solubles ('Brix) y la acidez no se ven afectados. Estos pretratamientos, también tienen un efecto protector sobre el color y la firmeza. A su vez, si se aplican niveles de presión altos por tiempos cortos se pueden inactivar enzimas y conservar el color (Denoya et al., 2017).

Otra fruta de interés, es el mango (Mangifera indica) el cual es muy apetecido por su aroma, sabor y alto valor nutritivo lo que hace importante garantizar una buena conservación de este producto vegetal. La aplicación de altas presiones en pulpa de mango (Kaushik et al., 2014) ha logrado conservar el color donde al ser procesado logra conservar un $85 \%$ del ácido ascórbico original, $92 \%$ de los fenoles totales y $90 \%$ de su capacidad antioxidante. Además de conservar el color también se pueden eliminar los coliformes presentes en la pulpa ya que son sensibles a las altas presiones. Al igual que el mango, la naranja (Citrus sinensis) también es una fruta muy apetecida por su alto contenido de nutrientes, combinada en ocasiones con zanahoria (Daucus carota) (Andrés et al., 2016) donde la aplicación de altas presiones permite inhibir el crecimiento microbiano reduciendo los recuentos de coliformes totales y los microorganismos sobrevivientes no tienden a aumentar significativamente en el transcurso del almacenamiento. En otro estudio, en pulpa de naranja (Buggenhout et al., 2015) evaluaron el efecto de la aplicación de la alta presión encontrando que mejoró la capacidad de retención de agua, la capacidad de hinchamiento y las propiedades reológicas. Los autores concluyeron que el tamaño de partícula está relacionado inversamente con la retención de agua. En cuanto a la fresa (Fragaria), otra fruta de gran interés comercial, se ha evaluado propiedades físicas (textura y firmeza), propiedades biofuncionales (fenoles totales, antocianinas totales, capacidad antioxidante) y la supervivencia de microorganismos en fresas tratadas con alta presión y con tratamiento térmico (Gao et al., 2016) evidenciando que si se almacenan a una temperatura de 4 ${ }^{\circ} \mathrm{C}$ tratadas con alta presión se conserva mejor la dureza, anhídridos totales, antocianinas totales y la capacidad antioxidante en comparación con el tratamiento térmico convencional. Por otra parte, el melón (Cucumis melo L) en combinación con otras frutas (Andrés et al., 2016), también ha sido tratado con altas presiones para evaluar como varían los sólidos solubles ('Brix), acidez titulable, vitamina C (ácido ascórbico), $\beta$-caroteno y el color. Obteniendo como resultado que no hubo efecto sobre los sólidos solubles ( ${ }^{\circ}$ Brix) y la acidez. Sin embargo, se presentó cambios en el color. En peras (Pyrus) mínimamente procesadas se ha aplicado altas presiones y $\mathrm{CO}_{2}$ para evaluar la inactivación de Saccharomyces cerevisiae (Valverde et al., 2010). Estas aplicaciones se han hecho a trozos de pera variando la presión $(6-30 \mathrm{MPa})$, temperatura $\left(25-55^{\circ} \mathrm{C}\right)$ y tiempo $(10-90 \mathrm{~min})$ de exposición sometiendo los trozos en contacto con dióxido de carbono comparándolo con tratamientos térmicos, siendo más eficaz la aplicación de altas presiones a una temperatura de $55^{\circ} \mathrm{C}$. Por otro lado el $\mathrm{pH}$ y los sólidos solubles ( $\left.{ }^{\circ} \mathrm{Brix}\right)$ no se vieron afectados por la alta presión.

Adicionalmente, en el Cuadro 1, se presentan los parámetros (variables de proceso) y el efecto de aplicación de esta tecnología en algunas frutas representativas. 
Cuadro 1. Parámetros y efecto de aplicaciones de APH en diferentes frutas.

\begin{tabular}{|c|c|c|c|c|c|}
\hline \multirow{2}{*}{ Fruta } & \multicolumn{3}{|c|}{ Parámetros de APH } & \multirow{2}{*}{ Efecto } & \multirow{2}{*}{ Referencia } \\
\hline & $\mathrm{P}(\mathrm{MPa})$ & $\mathrm{T}\left({ }^{\circ} \mathrm{C}\right)$ & $t(\min )$ & & \\
\hline $\begin{array}{l}\text { Durazno } \\
\text { (Prunus pérsica) }\end{array}$ & 400 & $21-35$ & 5 & $\begin{array}{l}\text { A mayor nivel de presión se puede inhibir el } \\
\text { pardeamiento de la fruta y disminuir la acti- } \\
\text { vidad residual de la enzima polifenoloxidasa. } \\
\text { Aumentando el tiempo de conservación. }\end{array}$ & $\begin{array}{l}\text { (Denoya et al., } \\
\text { 2015; Denoya et } \\
\text { al., 2016; Denoya } \\
\text { et al., 2017) }\end{array}$ \\
\hline $\begin{array}{l}\text { Mango } \\
\text { (Mangifera indica) }\end{array}$ & $100-600$ & $30 \pm 2$ & $1-20$ & $\begin{array}{l}\text { El pH disminuyó y la acidez titulable } \\
\text { aumentó favoreciendo la estabilidad de la } \\
\text { carga microbiana. El contenido de Vitamina } \\
\text { C (ácido ascórbico) y la capacidad antioxi- } \\
\text { dante aumento con el tratamiento de HAP. }\end{array}$ & $\begin{array}{l}\text { (Kaushik et al., } \\
\text { 2014) }\end{array}$ \\
\hline $\begin{array}{l}\text { Batido multifruta: } \\
\text { Naranja } \\
\text { (Citrus sinensis) } \\
\text { Papaya } \\
\text { (Carica papaya) } \\
\text { Melón } \\
\text { (Cucumis melo L) } \\
\text { Zanahoria } \\
\text { (Daucus carota) }\end{array}$ & $550-650$ & 20 & 3 & $\begin{array}{l}\text { Se presentó un incremento en el contenido } \\
\text { de carotenoides principalmente del licope- } \\
\text { no y el } \beta \text {-caroteno. Se presentó un efecto } \\
\text { positivo en la Vitamina C (ácido ascórbico) } \\
\text { y la actividad antioxidante (FRAP, DPPH), } \\
\text { presentando buena estabilidad durante el } \\
\text { almacenamiento. }\end{array}$ & $\begin{array}{l}\text { (Andrés et al., } \\
\text { 2016) }\end{array}$ \\
\hline $\begin{array}{l}\text { Fresa } \\
\text { (Fragaria) }\end{array}$ & 120 & 25 & 1 & $\begin{array}{l}\text { Se encontró mejor calidad y estabilidad } \\
\text { microbiológica en las muestras tratadas } \\
\text { con HAP almacenadas a } 4^{\circ} \mathrm{C} \text { respecto a las } \\
\text { de tratamiento térmico convencional. }\end{array}$ & (Gao et al., 2016) \\
\hline $\begin{array}{l}\text { Naranja } \\
\text { (Citrus sinensis) }\end{array}$ & $\begin{array}{l}80-150 \\
200-400\end{array}$ & 25 & $0,25-1$ & $\begin{array}{l}\text { Se obtuvo una suspensión de pulpa y jugo } \\
\text { de naranja más homogénea, con una mayor } \\
\text { capacidad de retención de agua y mejores } \\
\text { propiedades de hinchamiento y flujo. Se } \\
\text { incrementaron los compuestos bioactivos y } \\
\text { se mejoró la bioaccesibilidad. }\end{array}$ & $\begin{array}{l}\text { (Buggenhout et } \\
\text { al., 2015; Stinco } \\
\text { et al., 2020; De } \\
\text { Ancos et al., } \\
\text { 2020) }\end{array}$ \\
\hline $\begin{array}{l}\text { Chirimoya } \\
\text { (Annona cherimola) }\end{array}$ & 600 & $23-27$ & 8 & $\begin{array}{l}\text { El tratamiento con HAP disminuyó los } \\
\text { recuentos microbianos en cinco ciclos loga- } \\
\text { rítmicos. Representando una mayor calidad } \\
\text { microbiológica en la fruta. }\end{array}$ & $\begin{array}{l}\text { (Pérez-Pulido et } \\
\text { al., 2015) }\end{array}$ \\
\hline $\begin{array}{l}\text { Melón } \\
\text { (Cucumis melo L) }\end{array}$ & 600 & 25 & 10 & $\begin{array}{l}\text { No se alteraron los }{ }^{\circ} \text { Brix y la vitamina } C \\
\text { (ácido ascórbico) presentó una disminu- } \\
\text { ción, contraste los niveles de } \beta \text {-caroteno } \\
\text { fueron más altos. }\end{array}$ & $\begin{array}{l}\text { (Wolbang et al., } \\
\text { 2008) }\end{array}$ \\
\hline $\begin{array}{l}\text { Pera } \\
\text { (Pyrus) }\end{array}$ & 6-30 & $25-55$ & $10-90$ & $\begin{array}{l}\text { El pH y los }{ }^{\circ} \text { Brix no se vieron afectados. } \\
\text { Se presentó perdida de textura y oscure- } \\
\text { cimiento debido a una disminución de la } \\
\text { vitamina C (ácido ascórbico). La actividad } \\
\text { de la peroxidasa se redujo parcialmente. }\end{array}$ & $\begin{array}{l}\text { (Valverde et al., } \\
\text { 2010) }\end{array}$ \\
\hline
\end{tabular}

P(presión), T(temperatura), t (tiempo)

Jugos y zumos de frutas. La búsqueda de tecnologías innovadoras no térmicas (emergentes) para la producción de zumos y jugos de fruta seguros y de calidad es una tendencia reciente en la elaboración de este tipo de alimentos (Abid et al., 2014).

Por ejemplo, para un jugo de manzana (Malus domestica) es necesario el control de microorganismos, como también de enzimas que deterioran las características físicoquímicas del producto terminado, entre estas están: las polifeniloxidasas, peroxidasas y pectinmetilesterasas. Abid et al. (2014) reportaron que usando ultrasonido y altas presiones hidrostáticas a $450 \mathrm{MPa}$ por 10 minutos a $25^{\circ} \mathrm{C}$, se logra la inactivación total de enzimas alterantes y se mejoran las propiedades fisicoquímicas y antioxidantes. Bayindirli et al. (2006) evaluaron el proceso de aplicación de altas presiones en zumo de manzana y otros jugos, encontrando que el tratamiento que presentó mejores respuestas en cuanto a la reducción de actividad de la enzima polifenoloxidasa fue de $450 \mathrm{MPa}$ a 50 
${ }^{\circ} \mathrm{C}$ por 60 min, obteniendo una actividad residual de $9 \pm 2,25 \%$; en cuanto a las características microbiológicas de los productos tratados se presentó la inhibición de levaduras, mohos, Staphylococcus aureus, Escherichia coli O157: H7 y Salmonella. Yi et al. (2017) compararon el impacto en los cambios de calidad de zumos de manzana obtenidos a partir de tratamiento térmico convencional y de procesamiento por altas presiones $600 \mathrm{MPa}$ por 3 min; encontrando que los productos obtenidos por altas presiones fueron parecidos al zumo en fresco. Por otra parte, algunos microorganismos tienen la capacidad de deteriorar alimentos ácidos y producir micotoxinas. De manera específica, en el tratamiento de Ascosporas que son esporas resistentes a calores extremos y que contaminan jugos de manzana se utiliza la combinación de tratamientos térmicos y no térmicos para la disminución de estas. En tal sentido Evelyn and Silva (2016) utilizaron altas presiones de homogenización (HAP 600 MPa), ultrasonido de potencia (24 Khz) y tratamientos térmicos $\left(50,60,75^{\circ} \mathrm{C}\right)$ llegando a la conclusión de que la alta presión de homogenización es la mejor tecnología de conservación en la inactivación de esporas en el jugo de manzana. Una combinación de tratamientos no térmicos también puede atacar microorganismos no benéficos en jugos de manzana, siendo el caso de Alicyclobacillus acidoterrestris bacteria con muchas resistencias y que puede desarrollarse en jugos pasteurizados. Porębska et al. (2017) determinaron que el $\mathrm{CO}_{2}$ supercrítico y las altas presiones hidrostáticas a temperaturas moderadamente elevadas podrían ser una técnica útil para la inactivación de estas. Respecto a la vida útil de productos tratados con ultra altas presiones hidrostáticas (UAPH), Juarez-Enriquez et al. 82015) evaluaron lotes de jugo de manzana a 430 MPa durante 7 min, midiendo el efecto en la calidad fisicoquímica y microbiológica, durante el almacenamiento a $4{ }^{\circ} \mathrm{C}$ durante 34 días. La ultra alta presión hidrostática inactivó totalmente tanto la pectinmetilesterasa y la microbiota del jugo crudo, también se mantuvo el contenido de polifenoles y la capacidad antioxidante. Llegando a la conclusión de que se conservaron las propiedades fisicoquímicas y se obtuvo una buena calidad microbiológica.

En jugo de fresa (Fragaria), otro producto de interés agroindustrial, también se han realizado estudios empleando esta tecnología. Karacam et al. (2015) aplicaron altas presiones de homogenización a 60 y 100 MPa en diferentes ciclos para observar el efecto en la calidad de las propiedades físicas y químicas. Los autores notaron diferencias significativas en el contenido de sólidos solubles, a diferencia de la actividad antioxidante, y el contenido fenólico total que no presentaron diferencias significativas entre los tratamientos. Estos autores consideran que la alta presión de homogenización puede ser usada para el mejoramiento de propiedades físicas y químicas de los jugos de fresa, debido a que presentó un efecto positivo en el color dando como resultado un jugo deseable para los consumidores.

Por otra parte, en zumos de naranja (Citrus sinensis) los tratamientos con altas presiones se han utilizado debido al efecto positivo que presentan frente a los carotenoides y flavononas. Plaza et al. (2011) evaluaron el efecto de la aplicación de altas presiones de homogenización en zumo de naranja, encontrando aumentos significativos en contenidos totales de carotenoides y flavononas (45,19 y 15,46\% respectivamente en comparación al zumo no tratado (control). En cuanto a los compuestos bioactivos y actividad antioxidante en zumos de naranja (Velázquez-Estrada et al., 2012; Stinco et al., 2020) encontraron diferencias significativas entre la ultra alta presión de homogenización y la pasteurización, reportando que el zumo de naranja en fresco presentó el mismo valor nutricional en cuanto a la capacidad antioxidante que el tratado con ultra alta presión de homogenización mientras que el zumo pasteurizado presentó valores más bajos. A su vez, De Ancos et al. (2020) compararon también estos tratamientos encontrando que la aplicación de $300 \mathrm{MPa}$ en lotes de zumo de naranja permitió una reducción de la carga microbiana (Mesófilos, bacterias ácido lácticas, psicrotróficas y levaduras) en comparación con zumo pasteurizado. También se presentó una reducción de la actividad de la pectinmetilesterasa de hasta $96 \%$ de su actividad inicial. Evelyn and Filipa (2016) presentaron un estudio relacionado con el control de microorganismos presentes naturalmente en zumos de naranja, encontrando que el uso de las altas presiones $600 \mathrm{MPa}$ por $15 \mathrm{~min}$, mejoró la inactivación de esporas de Allicyclobacillus acidoterrestris usando como pretratamiento termosonificación a $78^{\circ} \mathrm{C}, 20,20 \mathrm{~W}$ en tiempos de 14 a $28 \mathrm{~min}$. Las levaduras y bacterias ácido lácticas también son microorganismos habituales como alterantes en zumo de naranja que disminuyen la vida útil del producto. Campos and Cristianini (2007) encontraron que usando alta presión de homogenización superior a 250 MPa en flujo continuo se lograron inactivar las cargas iniciales tanto de Saccharomyces cerevisiae como de Lactobacillus plantarum en lotes de zumo de naranja. Adicionalmente, Tahiri et al. (2006) evaluaron la inactivación de microorganismos patógenos en zumo de naranja; usando tecnología de altas presiones a $200 \mathrm{MPa}$, con 3 y 5 ciclos a 
temperatura ambiente, logrando la inactivación completa de Escherichia coli O157:H7. También informaron que el tratamiento fue más eficaz en cepas gram-negativas que en gram-positivas.

Los anteriores estudios, muestran el potencial que presenta esta tecnología emergente en la inactivación de microorganismos tales como: Escherichia coli, Lactobacillus plantarum, Leuconostoc mesenteroides, Saccharomyces cerevisiae, Penicillium spp, entre otros. Representando una alternativa eficiente para garantizar la inocuidad en zumos y jugos de fruta. Sin embargo, esta tecnología no solo ha sido aplicada en la inactivación de microorganismos y enzimas alterantes en zumos y jugos de fruta. También se ha utilizado en el control de compuestos bioactivos y otros atributos de calidad presentes en bebidas de frutas. Por ejemplo, en jugo de mango (Mangifera indica) (Guan et al., 2016) aplicaron altas presiones de homogenización encontrando que los carotenoides y la capacidad antioxidante fueron mayores en comparación con lotes tratados con tratamiento térmico convencional. Después de 60 días de almacenamiento el jugo presentó buenos atributos de calidad.

Para jugo de arándanos (Vaccinium macrocarpon), Barba et al. (2013) obtuvieron características similares en cuanto al control de los compuestos bioactivos, obteniendo una retención de hasta $92 \%$ de vitamina C (ácido ascórbico) e incremento de compuestos fenólicos y antocianinas totales con aplicación de altas presiones de $400 \mathrm{MPa}$ por $15 \mathrm{~min}$.

En jugo de sandía (Citrullus lanatus) compararon los efectos en los cambios de parámetros de calidad usando métodos convencionales y no convencionales. Observando que el tratamiento que menos afecto los atributos de calidad fue el de alta presión, en relación con el cambio de color, la viscosidad dinámica y concentración de licopeno (Zhang et al., 2011).

Laboissière et al. (2007) evaluaron la calidad sensorial del jugo de maracuyá (Passiflora edulis) tratado con altas presiones hidrostáticas comparándolo con el método de pasteurización convencional, llegando a la conclusión de que los parámetros obtenidos por altas presiones hidrostáticas son más semejantes a lotes de productos frescos en comparación a los jugos comerciales de esta fruta. Finalmente, Uckoo et al. (2013) evaluaron los parámetros de calidad en jugo de toronja (Citrus paradisi) tratados con altas presiones, tratamientos térmicos vs un control (fresco), encontrando que el tratamiento de altas presiones mantuvo los compuestos bioactivos en el jugo, concluyendo así que esta técnica es una alternativa viable en comparación con la pasteurización convencional de bebidas de frutas en fresco.

\section{Aplicación de otras tecnologías y perspectivas futuras}

La tecnología de las altas presiones y demás tecnologías emergentes, entre ellas: ultrasonido, calentamiento óhmico, plasma en frío, empaques 3D, entre otras. Sin lugar a duda, representan en la actualidad una alternativa de innovación para la agroindustria alimentaria en comparación con los tratamientos tradicionales de procesamiento. Debido a que estas tecnologías permiten no solo prolongar la vida útil de los productos, inactivar microorganismos y enzimas alterantes, sino también proteger a los compuestos bioactivos presentes en los alimentos mejorando así la calidad nutricional de los mismos. En tal sentido, los autores han adelantado una serie de investigaciones con el fin de evaluar los efectos de la aplicación de algunas de estas tecnologías tanto convencionales y no convencionales en la agroindustria de frutas y otros vegetales. Por ejemplo: Ordoñez-Santos et al. (2017) evaluaron el efecto de la aplicación de ultrasonido en jugo de uchuva (Physalis peruviana), encontrando un incremento final en los carotenoides, compuestos fenólicos y Provitamina A en lotes de jugo tratados con esta tecnología en comparación con el jugo pasteurizado, donde los resultados fueron inferiores. Ordoñez-Santos and Martínez-Girón (2020) informaron sobre la degradación cinética de compuestos bioactivos en jugo de tomate de árbol (Solanum betaceum) encontrando que el tratamiento térmico convencional a 70,80 y $90^{\circ} \mathrm{C}$ disminuyó significativamente los carotenoides y el contenido de Vitamina C (ácido ascórbico). Palacio-Vásquez et al. (2019) compararon diferentes tecnologías emergentes entre ellas: ultrasonido, microondas, fluidos supercríticos y enzimas para la extracción de glucósidos de stevia (Stevia rebaudiana), encontrando que todas las anteriores tecnologías son alternativas de extracción viables en la obtención de esteviósido y rebaudiósido A. 
Por otra parte, también se han adelantado estudios, que permitan el aprovechamiento de los subproductos de frutas. Ordoñez-Santos et al. (2019) estudiaron la viabilidad de la extracción de $\beta$-caroteno en epicarpio de chontaduro (Bactris gasipaes) mediante sonicación y aceite vegetal. Rivera-Ochoa et al. (2016) evaluaron el contenido de compuestos fenólicos totales y la actividad antioxidante en semilla y epicarpio de papaya (Carica papaya) encontrando que ambos subproductos representan un alto potencial agroindustrial. Shen et al. (2021) reportaron la extracción de los pigmentos responsables del color del epicarpio de jujube (Ziziphus jujuba) mediante la aplicación de la tecnología asistida por enzimas. Anwar et al. (2021) realizaron la extracción de polisacáridos solubles presentes en el epicarpio de malanga (Colocasia esculenta) mediante la aplicación de la tecnología de pulsos eléctricos y ultrasonido. Figueroa et al. (2021) reportaron el uso de la tecnología de extracción asistida por microondas en la extracción de polifenoles bioactivos con actividad antioxidante en epicarpio de aguacate (Persea americana Mill). Santoso et al. (2021) utilizaron la tecnología de plasma frío atmosférico para la reducción de tóxicos naturales en epicarpio de piña (Ananas comosus) sin afectar significativamente el contenido de azúcares fermentables. Zayed et al. (2021) reportaron el uso de diferentes tecnologías emergentes tales como: fluidos supercríticos, prensado en frío y ultrasonido en la extracción de compuestos bioactivos (fitoesteroles, carotenoides, tocoferoles, compuestos fenólicos, compuestos volátiles) a partir de semillas de cítricos como la mandarina (Citrus reticulata), pomelo (Citrus $\times$ paradisi) entre otras.

Dado lo anterior y teniendo en cuenta la importancia que representan las tecnologías emergentes para la agroindustria, las perspectivas e investigaciones futuras, deberían estar orientadas en:

1. Combinar el uso de las altas presiones con otras tecnologías emergentes ya existentes (ultrasonido, plasma frío, pulsos luminosos, campos magnéticos, entre otras) con el fin de evaluar los resultados en cuanto a la calidad final de diferentes productos agroalimentarios.

2. Desarrollar nuevas metodologías, técnicas, tecnologías emergentes aun no reportadas en la literatura que garanticen la calidad fisicoquímica, microbiológica, sensorial y nutricional del alimento durante su vida útil.

3. Desarrollar nuevos equipos amigables con el medio ambiente, fáciles de manipular, seguros, confiables y de baja inversión económica.

4. Realizar estudios de bioaccesibilidad y biodisponibilidad de compuestos bioactivos.

5. Desarrollar productos agroindustriales $5 \mathrm{~S}$ que respondan a las necesidades actuales de salud y nutrición.

6. Aprovechar la biomasa residual, como por ejemplo: subproductos que no hayan sido explorados desde el punto de vista de su potencial agroindustrial.

7. Desarrollar empaques comestibles y biopelículas degradables.

8. Preservar principios activos biológicos, nutracéuticos, entre otros; mediante el uso de tecnologías de barrera y aplicaciones biotecnológicas, como por ejemplo: microcápsulas o nanocápsulas.

9. Usar ingredientes naturales como sustitutos de aditivos sintéticos.

10. Realizar estudios de bioeconomía que permitan potenciar los agronegocios y la cadena de valor de los productos agroindustriales.

\section{CONCLUSIONES}

Elusodelatecnologíaemergentedealtaspresiones(incluyendoaltaspresioneshidrostáticasylahomogeneización por alta presión) son técnicas alternativas de conservación en frutas mínimamente procesadas, jugos y zumos, que favorecen las propiedades nutricionales y la inactivación de microorganismos y enzimas alterantes para ofrecer productos que conserven las características similares de las frutas en fresco y con una vida útil más prolongada, como respuesta a la demanda actual de los consumidores. En la aplicación de altas presiones en frutas mínimamente procesadas, jugos y zumos es importante evaluar primero la composición fisicoquímica de las frutas, debido a que esto influye en la determinación del tratamiento; en cuanto a la combinación de presión 
y tiempo, que permita lograr la inactivación de microorganismos y enzimas, sin afectar las características sensoriales y nutricionales del producto final durante su vida de anaquel.

\section{REFERENCIAS}

ABID,MUHAMMAD; JABBAR, SAQIB; HU,BING; HASHIM, MALIK-MUHAMMAD; WU, TAO; WU, ZHONGWEI; KHAN, MUHAMMAD-AMMAR; ZENG, XIAOXIONG. Synergistic impact of sonication and high hydrostatic pressure on microbial and enzymatic inactivation of apple juice. LWT-Food Science and Technology, v. 59, n. 1, 2014, p. 70-76. https://doi.org/10.1016/j.Iwt.2014.04.039

AGUDELO-MARTÍNEZ, PAOLA A.; LUNA-RAMÍREZ, JULIO C.; QUINTERO-CASTAÑO, VICTOR D. Formulación y evaluación fisicoquímica de jugo de mora (Rubus glaucus Benth) enriquecido con calcio y vitamina C. Biotecnología en el Sector Agropecuario y Agroindustrial, v. 18, n. 1, 2020, p. 56-63. https://doi.org/10.18684/bsaa.v18n1.1411

ANDRÉS, V.; MATEO-VIVARACHO, L.; GUILLAMÓN, E.; VILLANUEVA, M.J.; TENORIO, M.D. High hydrostatic pressure treatment and storage of soy-smoothies: Colour, bioactive compounds and antioxidant capacity. LWT-Food Science and Technology, v. 69, 2016, p. 123-130. https://doi.org/10.1016/j.Iwt.2016.01.033

ANWAR, MYLENE; BABU-GREESHMA; EL DIN, BEKHIT-ALAA. Utilization of ultrasound and pulse electric field for the extraction of water-soluble non-starch polysaccharide from taro (Colocasia esculenta) peel. Innovative Food Science and Emerging Technologies, v. 70, 2021, e102691. https://doi.org/10.1016/j.ifset.2021.102691

BARBA, FRANCISCO J.; ESTEVE, MARIA J.; FRIGOLA, ANA. Physicochemical and nutritional characteristics of blueberry juice after high pressure processing. Food Research International, v. 50, n. 2, 2013, p. 545-549. https://doi.org/10.1016/j.foodres.2011.02.038

BAYINDIRLI, ALEV; ALPAS, HAMI; BOZOGLU, FARUK; HIZAL, MIRZAHAN. Efficiency of high pressure treatment on inactivation of pathogenic microorganisms and enzymes in apple, orange, apricot and sour cherry juices. Food Control, v. 17, n. 1, 2006, p. 52-58. https://doi.org/10.1016/j.foodcont.2004.09.002

BOVER-CID, S.; BELLETTI, N.; GARRIGA, M.; AYMERICH, T. Model for Listeria monocytogenes inactivation on dry-cured ham by high hydrostatic pressure processing. Food Microbiology, v. 28, n. 4, 2011, p. 804-809. https://doi.org/10.1016/j.fm.2010.05.005

BUGGENHOUT, SANDY-VAN; WALLECAN, JOËL; CHRISTIAENS, STEFANIE; DEBON, STEPHANE J.J.; DESMET, CHRISTINA; LOEY, ANN-VAN; HENDRICKX, MARC; MAZOYER, JACQUES. Influence of high-pressure homogenization on functional properties of orange pulp. Innovative Food Science and Emerging Technologies, v. 30, 2015, p. 51-60. https://doi.org/10.1016/j.ifset.2015.05.004

CALLIGARIS, SONIA; FOSCHIA, MARTINA; BARTOLOMEOLI, INGRID; MAIFRENI, MICHELA; MANZOCCO, LARA. Study on the applicability of high-pressure homogenization for the production of banana juices. LWT-Food Science and Technology, v. 45, n. 1, 2012, p. 117-121. https://doi.org/10.1016/j.lwt.2011.07.026

CAMPOS, F.P.; CRISTIANINI, M. Inactivation of Saccharomyces cerevisiae and Lactobacillus plantarum in orange juice using ultra high-pressure homogenisation. Innovative Food Science and Emerging Technologies, v. 8, 2007, p. 226-229. https://doi.org/10.1016/j.ifset.2006.12.002

CHENG, CHUAN-XIANG; JIA, MENG; GUI, YAO; MA, YAQIN. Comparison of the effects of novel processing technologies and conventional thermal pasteurisation on the nutritional quality and aroma of Mandarin (Citrus unshiu) juice. Innovative Food Science \& Emerging Technologies, v. 64, 2020, e102425.

https://doi.org/10.1016/j.ifset.2020.102425 
DAI, JINMING; BAI, MEI; LI, CHANGZHU; CUI, HAIYING; LIN, LIN. Advances in the mechanism of different antibacterial strategies based on ultrasound technique for controlling bacterial contamination in food industry. Trends in Food Science \& Technology, v. 105, 2020, p. 211-222. https://doi.org/10.1016/j.tifs.2020.09.016

DE ANCOS, BEGOÑA; RODRIGO, MARÍA J.; SÁNCHEZ-MORENO, CONCEPCIÓN; CANO, M. PILAR; ZACARÍAS, LORENZO. Effect of high-pressure processing applied as pretreatment on carotenoids, flavonoids and vitamin $\mathrm{C}$ in juice of the sweet oranges Navel and the red-fleshed Cara. Food Research International, v. 132, 2020, e109105. https://doi.org/10.1016/j.foodres.2020.109105

DENOYA, G.I.; POLENTA, G.A.; APÓSTOLO, N.M.; BUDDE, C.O.; SANCHO, A.M.; VAUDAGNA, S.R. Optimization of high hydrostatic pressure processing for the preservation of minimally processed peach pieces. Innovative Food Science and Emerging Technologies, v. 33, 2015, p. 84-93. https://doi.org/10.1016/j.ifset.2015.11.014

DENOYA, G.I.; NANNI, M.S.; APÓSTOLO, N.M.; VAUDAGNA, S.R.; POLENTA, G.A. Biochemical and microstructural assessment of minimally processed peaches subjected to high-pressure processing: Implications on the freshness condition. Innovative Food Science and Emerging Technologies, v. 36, 2016, p. 212-220. https://doi.org/10.1016/j.ifset.2016.06.026

DENOYA, G.I., VAUDAGNA, SERGIO R.; CHAMORRO, VERÓNICA C.; GODOY, M. FERNANDA; BUDDE, CLAUDIO O.; POLENTA, GUSTAVO A. Suitability of different varieties of peaches for producing minimally processed peaches preserved by high hydrostatic pressure and selection of process parameters. LWT-Food Science and Technology, v. 78, 2017, p. 367-372. https://doi.org/10.1016/j.lwt.2017.01.006

DESHAWARE, S.; GUPTA, S.; SINGHAL, R.; VARIYAR, P.S. Influence of different pasteurization techniques on antidiabetic, antioxidant and sensory quality of debittered bitter gourd juice during storage. Food Chemistry, v. 285, 2019, p. 156-162. https://doi.org/10.1016/j.foodchem.2019.01.140

DUMAY, ELIANE; CHEVALIER-LUCIA, DOMINIQUE; PICART-PALMADE, LAËTITIA; BENZARIA, AMAL; GRÀCIA-JULIÀ, ALVAR; BLAYO, CLAIRE. Technological aspects and potential applications of (ultra) high-pressure homogenisation. Trends in Food Science and Technology, v. 31, n. 1, 2013, p.13-26. https://doi.org/10.1016/j.tifs.2012.03.005

EVELYN, KIM, H.J.; SILVA, F.V.M. Modeling the inactivation of Neosartorya fischeri ascospores in apple juice by high pressure, power ultrasound and thermal processing. Food Control, v. 59, 2016, p. 530-537 https://doi.org/10.1016/j.foodcont.2015.06.033

EVELYN, SILVA; FILIPA V.M. High pressure processing pretreatment enhanced the thermosonication inactivation of Alicyclobacillus acidoterrestris spores in orange juice. Food Control, v. 62, 2016, p. 365-372. https://doi.org/10.1016/j.foodcont.2015.11.007

FIGUEROA, JORGE G.; BORRAS-LINARES, ISABEL; DEL PINO-GARCÍA, RAQUEL; CURIEL, JOSÉ ANTONIO; LOZANO-SANCHEZ, JESÚS; SEGURA-CARRETERO, ANTONIO. Functional ingredient from avocado peel: Microwave-assisted extraction, characterization and potential applications for the food industry. Food Chemistry, v. 352, 2021, e129300. https://doi.org/10.1016/j.foodchem.2021.129300

GAO, GE; REN, PENGYAN; CAO, XIAMIN; YAN, BING; LIAO, XIAOJUN; SUN, ZHIJIAN; WANG, YONGTAO. Comparing quality changes of cupped strawberry treated by high hydrostatic pressure and thermal processing during storage. Food and Bioproducts Processing, v. 100, 2016, p. 221-229. https://doi.org/10.1016/j.fbp.2016.06.017

GHARIBZAHEDI-TAGHI, MOHAMMAD-SEYED; HERNÁNDEZ-ORTEGA, CÉSAR; WELTI-CHANES, JORGE; PUTNIK, PREDRAG; BARBA, FRANCISCO J.; MALLIKARJUNAN, KUMAR; ESCOBEDO-AVELLANEDA, ZAMANTHA; ROOHINEJAD, SHAHIN. High pressure processing of food-grade emulsion systems: Antimicrobial activity, and effect on the physicochemical properties. Food Hydrocolloids, v. 87, 2019, p. 307-320. https://doi.org/10.1016/j.foodhyd.2018.08.012 
GIRALDO-GIL, ALEXANDER; OCHOA-GONZÁLEZ, OSCAR-ALBERTO; CARDONA- SEPÚLVEDA, LUIS-FERNANDO; ALVARADO-TORRES, PEDRO-NEL. Venting stage experimental study of food sterilization process in a vertical retort using temperature distribution tests and energy balances. Case Studies in Thermal Engineering, v. 22, 2020, e100736. https://doi.org/10.1016/j.csite.2020.100736

GÓMEZ-MAQUEO, ANDREA; WELTI-CHANES, JORGE; PILAR-CANO, M. Release mechanisms of bioactive compounds in fruits submitted to high hydrostatic pressure: A dynamic microstructural analysis based on prickly pear cells. Food Research International, v. 130, 2020, e108909. https://doi.org/10.1016/j.foodres.2019.108909

GUAN,YUNJING; ZHOU, LINYAN; BI, JINFENG; YI, JIANYONG; LIU, XUAN; CHEN, QINQIN; WU, XINYE; ZHOU, M.O. Change of microbial and quality attributes of mango juice treated by high pressure homogenization combined with moderate inlet temperatures during storage. Innovative Food Science and Emerging Technologies, v. 36, 2016, p. 320-329. https://doi.org/10.1016/j.ifset.2016.07.009

HRADECKY, J.; KLUDSKA, E.; BELKOVA, B.; WAGNER, M.; HAJSLOVA, J. Ohmic heating: A promising technology to reduce furan formation in sterilized vegetable and vegetable/meat baby foods. Innovative Food Science \& Emerging Technologies, v. 43, 2017, p.1-6. https://doi.org/10.1016/j.ifset.2017.07.018

HU, KAI; PENG, D.A.; WANG, LAN; LIU, HAO; XIE, BIJUN; SUN, ZHIDA. Effect of mild high hydrostatic pressure treatments on physiological and physicochemical characteristics and carotenoid biosynthesis in postharvest mango. Postharvest Biology and Technology, v. 172, 2021, e111381. https://doi.org/10.1016/j.postharvbio.2020.111381

HUANG, HSIAO-WEN; HSU, CHIAO-PING; WANG, CHUNG-YI. Healthy expectations of high hydrostatic pressure treatment in food processing industry. Journal of Food and Drug Analysis, v. 28, n. 1, 2020, p.1-13. https://doi.org/10.1016/j.jfda.2019.10.002

JIN, SHUGUANG; ZHANG, GUANGMING; ZHANG, PANYUE; FAN, SHIYANG; LI, FAN. High-pressure homogenization pretreatment of four different lignocellulosic biomass for enhancing enzymatic digestibility. Bioresource Technology, v. 181, 2015, p. 270-274. https://doi.org/10.1016/j.biortech.2015.01.069

JUAREZ-ENRIQUEZ, E.; SALMERON-OCHOA, I.; GUTIERREZ-MENDEZ, N.; RAMASWAMY, H.S.; ORTEGA-RIVAS, E. Shelf life studies on apple juice pasteurised by ultrahigh hydrostatic pressure. LWT-Food Science and Technology, v. 62, n. 1, 2015, p. 915-919. https://doi.org/10.1016/j.lwt.2014.07.041

KARACAM, CAGRI-HELIN; SAHIN, SERPIL; OZTOP, MECIT-HALIL. Effect of high pressure homogenization (microfluidization) on the quality of Ottoman Strawberry (F. Ananassa) juice. LWT-Food Science and Technology, v. 64, 2015, p. 932-937. https://doi.org/10.1016/j.Iwt.2015.06.064

KAUSHIK, NEELIMA; KAUR, BARJINDER-PAL; RAO, P. SRINIVASA; MISHRA, H.N. Effect of high pressure processing on color, biochemical and microbiological characteristics of mango pulp (Mangifera indica cV. Amrapali). Innovative Food Science and Emerging Technologies, v. 22, 2014, p. 40-50. https://doi.org/10.1016/j.ifset.2013.12.011

LABOISSIĖRE, L.H.E.S; DELIZA, R; BARROS-MARCELLINI, A.M.; ROSENTHAL, A.; CAMARGO, L.M.A.Q.; JUNQUEIRA, R.G. Effects of high hydrostatic pressure (HHP) on sensory characteristics of yellow passion fruit juice. Innovative Food Science and Emerging Technologies, v. 8, n. 4, 2007, p. 469-477. https://doi.org/10.1016/j.ifset.2007.04.001

LIU, HSIAO-HUI; KUO, MENG-I. Ultra high pressure homogenization effect on the proteins in soy flour. Food Hydrocolloids, v. 52, 2016, p. 741-748.

https://doi.org/10.1016/j.foodhyd.2015.08.018 
MANASSERO, CARLOS A.; SPERONI, FRANCISCO; VAUDAGNA, SERGIO R. High hydrostatic pressure treatment improves physicochemical properties of calcium- and soybean protein-added peach juice. LWT- Food Science and Technology, v. 101, 2019, p. 54-60. https://doi.org/10.1016/j.lwt.2018.11.021

MANIGLIA, BIANCA C.; CASTANHA, NANCI; ROJAS, MELIZA-LINDSAY; AUGUSTO, PEDRO E.D. Emerging technologies to enhance starch performance. Current Opinion in Food Science, v. 37, 2021, p. 26-36. https://doi.org/10.1016/j.cofs.2020.09.003

MARCOS, BEGONYA; AYMERICH, TERESA; GUARDIA, M DOLORS, GARRIGA, MARGARITA. Assessment of high hydrostatic pressure and starter culture on the quality properties of low-acid fermented sausages. Meat Science, v. 76, n. 1, 2007, p. 46-53. https://doi.org/10.1016/j.meatsci.2006.09.020

MARESCA, PAOLA; DONSİ, FRANCESCO; FERRARI, GIOVANNA. Application of a multi-pass high-pressure homogenization treatment for the pasteurization of fruit juices. Journal of Food Engineering, v. 104, n. 3 , 2011, p. 364-372. https://doi.org/10.1016/j.jfoodeng.2010.12.030

MARTÍN-VERTEDOR, DANIEL; RODRIGUES, NUNO; MARX, ITALA M.G.; VELOSO, ANA C.A.; PERES, ANTÓNIO M.; PEREIRA, JOSÉ-ALBERTO. Impact of thermal sterilization on the physicochemical-sensory characteristics of Californian-style black olives and its assessment using an electronic tongue. Food Control, v. 117, 2020, e107369. https://doi.org/10.1016/j.foodcont.2020.107369

MENG, LING; ZHANG, WENCHENG; ZHOU, XIANHAN; WU, ZEYU; HUI, AILING; HE, YIWEN., GAO, HAN; CHEN, PENGPENG. Effect of high hydrostatic pressure on the bioactive compounds, antioxidant activity and in vitro digestibility of cooked black rice during refrigerated storage. Journal of Cereal Science, v. 86, 2019, p. 54-59. https://doi.org/10.1016/j.jcs.2019.01.005

MIGUEL-PINTADO, CRISTINA; NOGALES, SERGIO; FERNÁNDEZ-LEÓN, ANA M.; DELGADO-ADÁMEZ, JONATHAN; HERNÁNDEZ, TERESA; LOZANO, MERCEDES; CAÑADA-CAÑADA, FLORENTINA; RAMÍREZ, ROSARIO. Effect of hydrostatic high pressure processing on nectarine halves pretreated with ascorbic acid and calcium during refrigerated storage. LWT-Food Science and Technology, v. 54, n.1, 2013, p. 278-284. https://doi.org/10.1016/j.Iwt.2013.05.026

ORDÓÑEZ-SANTOS, LUIS-EDUARDO; MARTÍNEZ-GIRÓN, JADER; ARIAS-JARAMILLO, MARIA-ENITH. Effect of ultrasound treatment on visual color, vitamin C, total phenols, and carotenoids content in Cape gooseberry juice. Food Chemistry, v. 233, 2017, p. 96-100. https://doi.org/10.1016/j.foodchem.2017.04.114

ORDÓÑEZ-SANTOS, LUIS-EDUARDO., MARTÍNEZ-GIRÓN, JADER. Thermal degradation kinetics of carotenoids, vitamin $C$ and provitamin $A$ in tree tomato juice. International Journal of Food Science and Technology, v. 55, 2020, p. 201-210. https://doi.org/10.1111/ijfs.14263

ORDÓÑEZ-SANTOS, LUIS-EDUARDO., MARTÍNEZ-GIRÓN, JADER.; RODRÍGUEZ-RODRÍGUEZ, DIANA-XIMENA. Extraction of total carotenoids from peach palm fruit (Bactris gasipaes) peel by means of ultrasound application and vegetable oil. DYNA, v. 86, n. 209, 2019, p. 98-103. https://doi.org/10.15446/dyna.v86n209.74840

PALACIO-VASQUEZ, ESTEBAN; ARROYAVE-ROA, JUAN-DIEGO; CARDONA-CAICEDO, MAURICIO; HURTADO-IBARBO, JOHN-HEVERTH., MARTÍNEZ-GIRÓN, JADER. Extracción de glucósidos de stevia rebaudiana (bertoni) a partir de tecnologías de extracción verdes. Revista de Investigación Agraria y Ambiental, v. 10, n.1, 2019, p. 43-56.

https://doi.org/10.22490/21456453.2336 
PERDOMO-LAMILLA, CAROLINA; VAUDAGNA, SERGIO R.; CAP, MARIANA; RODRIGUEZ, ANABEL. Application of high pressure-assisted infusion treatment to mango pieces: Effect on quality properties. Innovative Food Science and Emerging Technologies, v. 64, 2020, e102431. https://doi.org/10.1016/j.ifset.2020.102431

PÉREZ-PULIDO, RUBÉN; TOLEDO, JULIA; GRANDE, M. JOSÉ; GÁLVEZ, ANTONIO; LUCAS, ROSARIO. Analysis of the effect of high hydrostatic pressure treatment and enterocin AS-48 addition on the bacterial communities of cherimoya pulp. International Journal of Food Microbiology, v. 196, 2015, p. 62-69. https://doi.org/10.1016/j.ijfoodmicro.2014.11.033

PLAZA, LUCÍA; SÁNCHEZ-MORENO, CONCEPCIÓN; DE ANCOS, BEGOÑA; ELEZ-MARTÍNEZ, PEDRO; MARTÍN-BELLOSO, OLGA; CANO, M. PILAR. Carotenoid and flavanone content during refrigerated storage of orange juice processed by high-pressure, pulsed electric fields and low pasteurization. LWT-Food Science and Technology, v. 44, n. 4, 2011, p. 834-839. https://doi.org/10.1016/j.Iwt.2010.12.013

PORĘBSKA, IZABELA; SOKOŁOWSKA, BARBARA; SKĄPSKA, SYLWIA; RZOSKA, SYLWESTER J. Treatment with high hydrostatic pressure and supercritical carbon dioxide to control Alicyclobacillus acidoterrestris spores in apple juice. Food Control, v. 73, 2017, p. 24-30. https://doi.org/10.1016/j.foodcont.2016.06.005

RIOS-CORRIPIO, GABRIELA; WELTI-CHANES, JORGE; RODRÍGUEZ-MARTÍNEZ, VERÓNICA; GUERRERO-BELTRÁN, JOSÉ-ÁNGEL. Influence of high hydrostatic pressure processing on physicochemical characteristics of a fermented pomegranate (Punica granatum L.) beverage. Innovative Food Science and Emerging Technologies, v. 59, 2020, e102249. https://doi.org/10.1016/j.ifset.2019.102249

RIVERA-OCHOA, M.C.; CASTILLO-ROBLES, N.Z.; FIGUEROA-SEPÚLVEDA, K.; ROJAS- DORADO, M.C.; ESPARZA, J.; ORDOÑEZ-SANTOS, L.E. Determinación de los compuestos fenólicos totales y actividad antioxidante en residuos de fruto de papaya (Carica papaya). Vitae, v. 23, supl. 1, 2016, p. 70-73.

RUIZ, V.; ALONSO, R.; SALVADOR, M.; CONDÓN, S.; CONDÓN-ABANTO, S. Impact of shoulders on the calculus of heat sterilization treatments with different bacterial spores. Food Microbiology, v. 94, 2021, e103663. https://doi.org/10.1016/j.fm.2020.103663

SANTOSO, SHELLA-PERMATASARI; LIN, SHIN-PING; WANG, TAN-YING; TING, YUWEN; HSIEH, CHANGWEI; YU, ROCH-CHUI; ANGKAWIJAYA, ARTIK-ELISA; SOETAREDJO, FELYCIA-EDI; HSU, HSIEN-YI; CHENG, KUAN-CHEN. Atmospheric cold plasma-assisted pineapple peel waste hydrolysate detoxification for the production of bacterial cellulose. International Journal of Biological Macromolecules, v. 175, 2021, p. 526-534.

https://doi.org/10.1016/j.ijbiomac.2021.01.169

SHEN, DONGBEI; KOU-XIAOHONG; WU-CAIE; FAN-GONGJIAN; LI-TINGTING; DOU- JINFENG; WANG-HANBO; ZHU-JINPENG. Cocktail enzyme-assisted alkaline extraction and identification of jujube peel pigments. Food Chemistry, v. 357, 2021, e129747.

https://doi.org/10.1016/j.foodchem.2021.129747

STINCO, CARLA M.; SENTANDREU, ENRIQUE; MAPELLI-BRAHM, PAULA; NAVARRO, JOSÉ L.; VICARIO, ISABEL M.; MELÉNDEZ-MARTÍNEZ, ANTONIO J. Influence of high pressure homogenization and pasteurization on the in vitro bioaccessibility of carotenoids and flavonoids in orange juice. Food Chemistry, v. 331, 2020, e127259. https://doi.org/10.1016/j.foodchem.2020.127259

SUÁREZ-JACOBO, ÁNGELA; RÜFER, CORINNA E.; GERVILLA, RAMÓN; GUAMIS, BUENAVENTURA; ROIGSAGUÉS, ARTUR X.; SALDO, JORDI. Influence of ultra-high pressure homogenisation on antioxidant capacity, polyphenol and vitamin content of clear apple juice. Food Chemistry, v. 127, n. 2, 2011, p. 447-454 https://doi.org/10.1016/j.foodchem.2010.12.152

TAHIRI, IMANE; MAKHLOUF, JOSEPH; PAQUIN, PAUL; FLISS, ISMAIL. Inactivation of food spoilage bacteria and Escherichia coli O157: H7 in phosphate buffer and orange juice using dynamic high pressure. Food Research International, v. 39, n. 1, 2006, p.98-105.

https://doi.org/10.1016/j.foodres.2005.06.005 
UCKOO, RAM M.; JAYAPRAKASHA, GUDDADARANGAVVANAHALLY K.; SOMERVILLE, JEREMY A.; BALASUBRAMANIAM, V.M.; PINARTE, MONICA; PATIL, BHIMANAGOUDA S. High pressure processing controls microbial growth and minimally alters the levels of health promoting compounds in grapefruit (Citrus paradisi Macfad) juice. Innovative Food Science and Emerging Technologies, v. 18, 2013, p. 7-14. https://doi.org/10.1016/j.ifset.2012.11.010

UNITED STATES DEPARTMENT OF AGRICULTURE (USDA). High pressure processing (HPP) and inspection program personnel (IPP) verification responsibilities. FSIS Directive, 6120.1, 2012, p. 1-8.

VALVERDE, M.T.; MARÍN-INIESTA, F.; CALVO, L. Inactivation of Saccharomyces cerevisiae in conference pear with high pressure carbon dioxide and effects on pear quality. Journal of Food Engineering, v. 98, n. 4, 2010, p. 421-428. https://doi.org/10.1016/j.jfoodeng.2010.01.022

VELÁZQUEZ-ESTRADA, R.M.; HERNÁNDEZ-HERRERO, M.M.; GUAMIS-LÓPEZ, B.; ROIG-SAGUÉS, A.X. Impact of ultra high pressure homogenization on pectin methylesterase activity and microbial characteristics of orange juice: A comparative study against conventional heat pasteurization. Innovative Food Science and Emerging Technologies, v. 13, 2012, p.100-106. https://doi.org/10.1016/j.ifset.2011.09.001

WOLBANG, CARLA M.; FITOS, JACQUELINE L.; TREEBY, MICHAEL T. The effect of high pressure processing on nutritional value and quality attributes of Cucumis melo L. Innovative Food Science and Emerging Technologies, v. 9, n. 2, 2008, p. 196-200. https://doi.org/10.1016/j.ifset.2007.08.001

YE, RAN; HARTE, FEDERICO. High pressure homogenization to improve the stability of casein-hydroxypropyl cellulose aqueous systems. Food Hydrocolloids, v. 35, 2014, p.670-677. https://doi.org/10.1016/j.foodhyd.2013.08.022

YI, JUNJIE; KEBEDE, BINIAM T.; DANG-DOAN, NGOC-HAI; BUVÉ, CAROLIEN; GRAUWET,TARA; LOEY, ANNVAN; HU, XIAOSONG; HENDRICKX, MARC. Quality change during high pressure processing and thermal processing of cloudy apple juice. LWT-Food Science and Technology, v. 75, 2017, p. 85-92. https://doi.org/10.1016/j.Iwt.2016.08.041

ZAYED, AHMED; BADAWY, MARWA T.; FARAG, MOHAMED A. Valorization and extraction optimization of Citrus seeds for food and functional food applications. Food Chemistry, v. 355, 2021, e129609. https://doi.org/10.1016/j.foodchem.2021.129609

ZHANG, CHAO; TRIERWEILER, BERNHARD; LI, WU; BUTZ, PETER; XU,YONG; RÜFER, CORINNA E.; MA, YUE; ZHAO, XIAOYAN. Comparison of thermal, ultraviolet-c, and high pressure treatments on quality parameters of watermelon juice. Food Chemistry, v. 126, n. 1, 2011, p. 254-260. https://doi.org/10.1016/j.foodchem.2010.11.013 